\title{
X-ray spectral evolution of TeV BL Lacertae objects: eleven years of observations with BeppoSAX, XMM-Newton and Swift satellites (Corrigendum)
}

\author{
F. Massaro ${ }^{1}$, A. Tramacere ${ }^{2}$, A. Cavaliere ${ }^{1}$, M. Perri ${ }^{3}$, and P. Giommi ${ }^{3}$ \\ ${ }^{1}$ Dipartimento di Fisica, Università di Roma Tor Vergata via della Ricerca scientifica 1, 00133 Roma, Italy \\ e-mail: fmassaro@cfa.harvard.edu \\ 2 Dipartimento di Fisica, Università di Roma La Sapienza, Piazzale A. Moro 2, 00185 Roma, Italy \\ 3 ASI Science Data Center, ESRIN, 00044 Frascati, Italy
}

A\&A 478, 395-401 (2008), DOI: 10.1051/0004-6361:20078639

Key words. galaxies: active - BL Lacertae objects: general - X-rays: galaxies - radiation mechanisms: non-thermal - errata, addenda

Due to an error in reporting the spectral parameters of Mrk 421 from literature, the upper panels of Figs. 1, 2, 4, 6, 8 have a mistake. In these figures, the height of the SED at the peak energy for Mrk 421 was incorrectly reported from the literature (see Tramacere, A., Massaro, F., \& Cavaliere, A., 2007, A\&A, 466, $521)$ as being a factor of 10 lower than it's true value. However, the conclusions and the results of the paper are not affected by the above mistake. Here in the following there are the corrected version of the above figures.

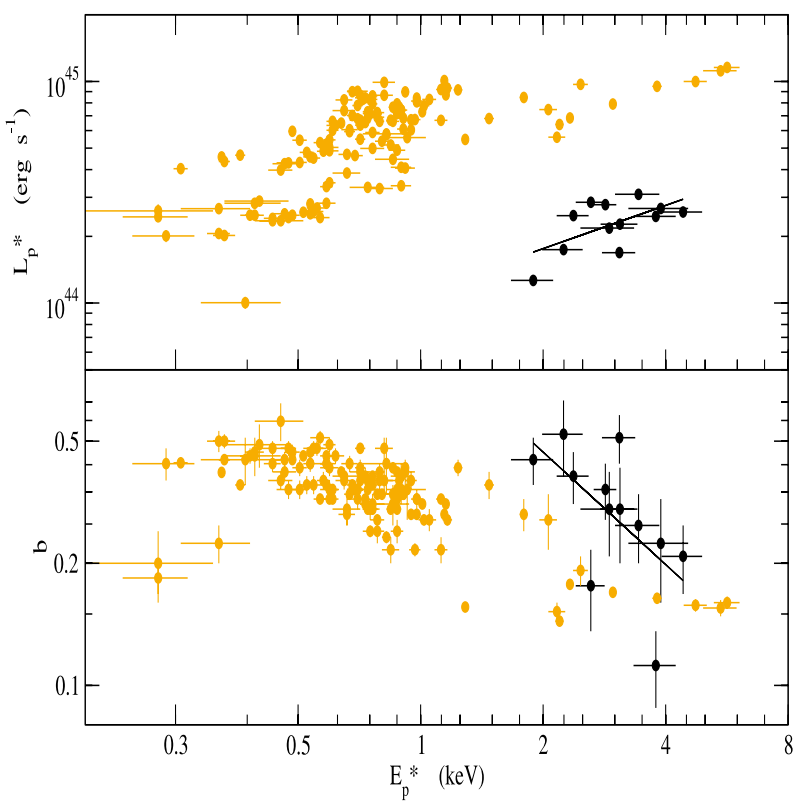

Fig. 1. $E_{\mathrm{p}}^{*}-L_{\mathrm{p}}^{*}$ and $E_{\mathrm{p}}^{*}-b$ plots for PKS 0548-322 (black filled squares) compared with those of Mrk 421 (orange circles). Black lines indicate the regressions underlying the $r_{\log }$ correlation coefficient.

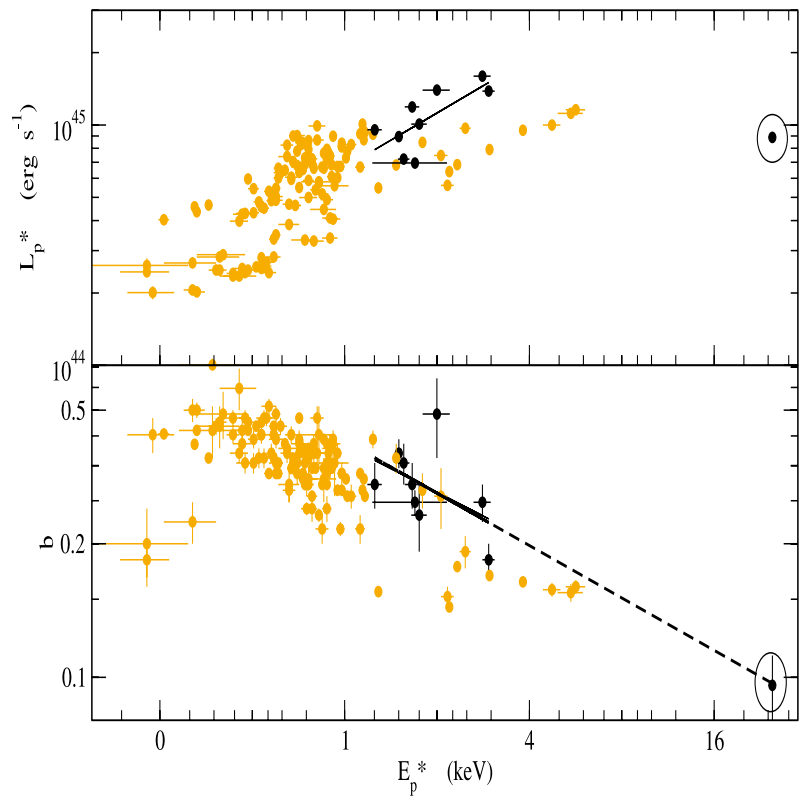

Fig. 2. $E_{\mathrm{p}}^{*}-L_{\mathrm{p}}^{*}$ and $E_{\mathrm{p}}^{*}-b$ plots for $1 \mathrm{H} 1426+428$ (black filled squares) compared with those of Mrk 421 (orange circles). Black lines indicate the regressions underlying the $r_{\log }$ correlation coefficient. Circled values refers to the peculiar observation performed on the 16 June 2001 by XMM-Newton (see Massaro et al. 2008, A\&A, 478, 395, for more details). 
A\&A 528, C1 (2011)

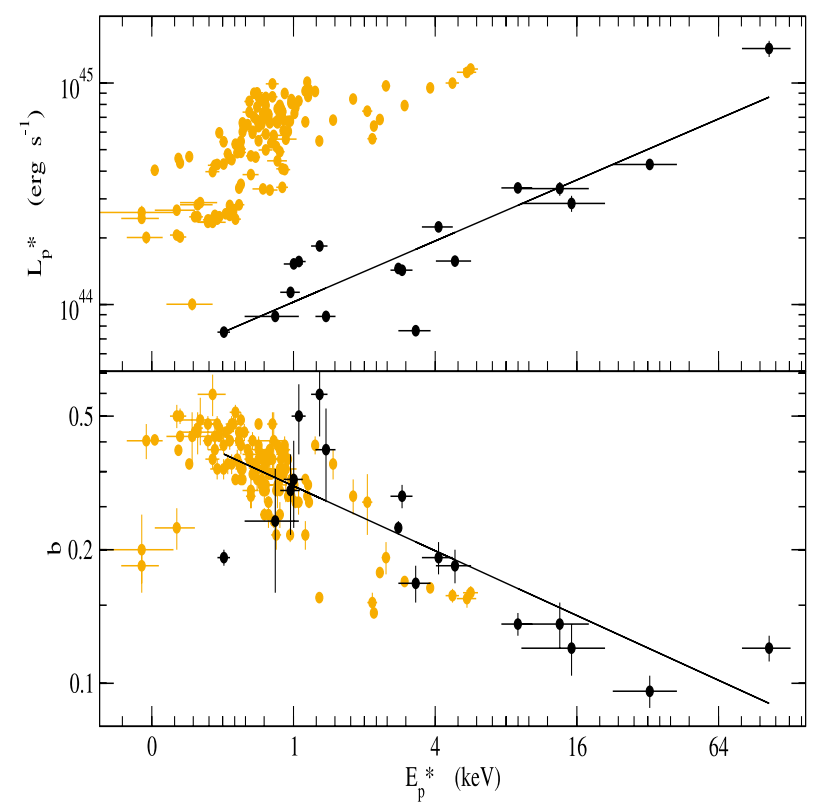

Fig. 3. $E_{\mathrm{p}}^{*}-L_{\mathrm{p}}^{*}$ and $E_{\mathrm{p}}^{*}-b$ plots for Mrk 501 (black filled squares) compared with those of Mrk 421 (orange circles). Black lines indicate the regressions underlying the $r_{\log }$ correlation coefficient.

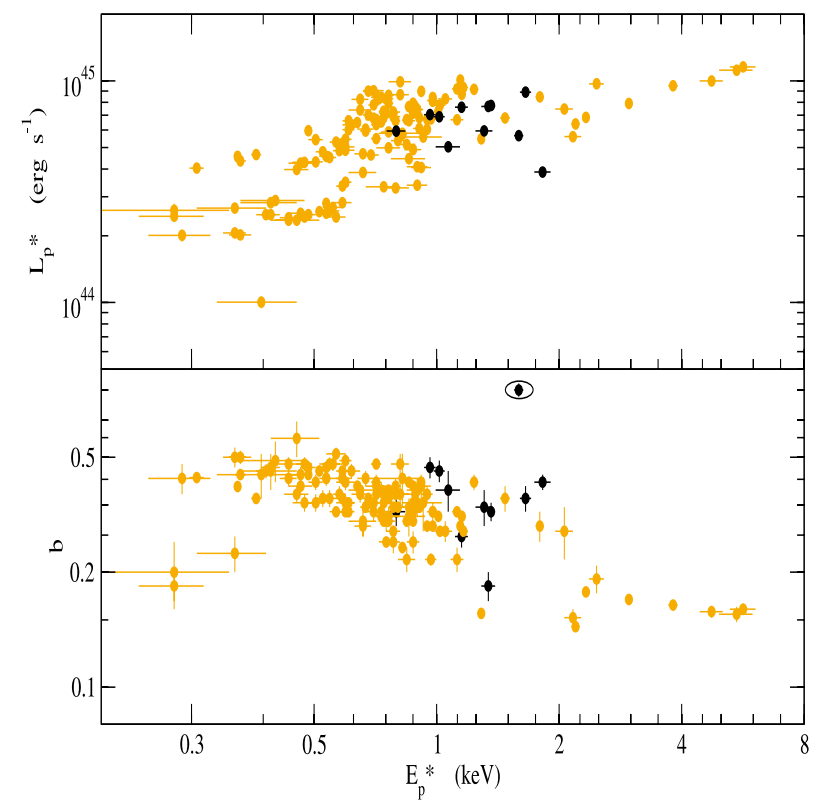

Fig. 4. $E_{\mathrm{p}}^{*}-L_{\mathrm{p}}^{*}$ and $E_{\mathrm{p}}^{*}-b$ plots for $1 \mathrm{ES} 1959+650$ (black filled squares) compared with those of Mrk 421 (orange circles). Circled values refers to the peculiar observation performed on the 29 May 2006 by Swift (see Massaro et al. 2008, A\&A, 478, 395, for more details).

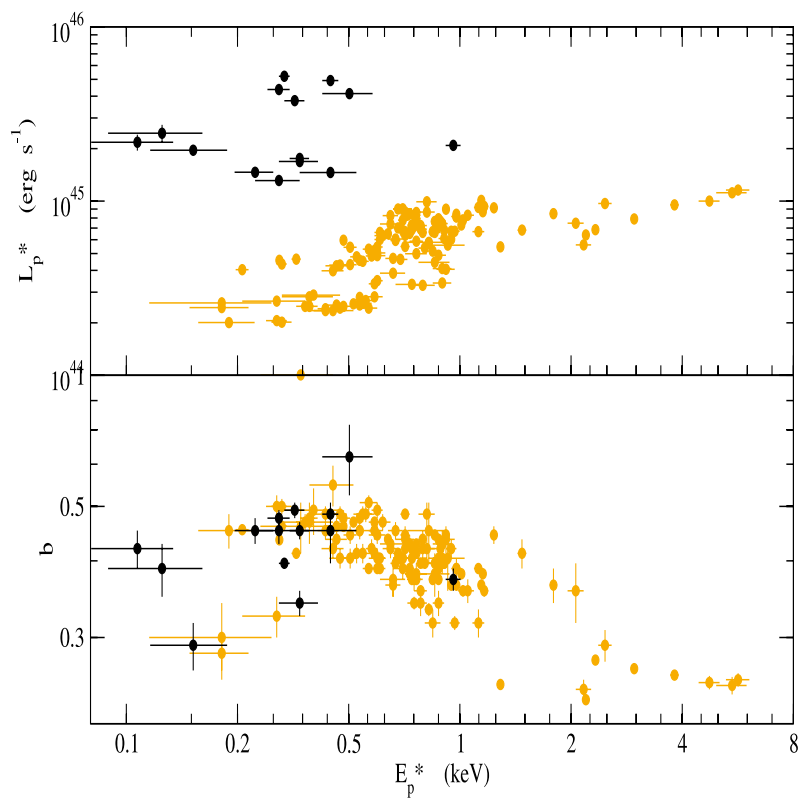

Fig. 5. $E_{\mathrm{p}}^{*}-L_{\mathrm{p}}^{*}$ and $E_{\mathrm{p}}^{*}-b$ plots for PKS 2155-304 (black filled squares) compared with those of Mrk 421 (orange circles). 